\title{
Ergoespirometria. Teste de Esforço Cardiopulmonar, Metodologia e Interpretação
}

\author{
Paulo Yazbek Jr, Ricardo Tavares de Carvalho, Lívia Maria dos Santos Sabbag, \\ Linamara Rizzo Battistella
}

São Paulo, SP

O teste ergométrico (TE) convencional, com interpretação do eletrocardiograma, apresenta-se como um método valioso para detectar modificações entre a disponibilidade e a necessidade de oxigênio pelo miocárdio, avaliar arritmias e o comportamento da pressão arterial com ou sem o uso de medicamentos. Porém, muitas questões da função cardiovascular e respiratória ficam sem resposta diante de uma avaliação pelo $\mathrm{TE}^{1-5}$.

O diagnóstico e avaliação de insuficiência cardíaca em uma extremidade, e a real capacidade funcional de atletas em outro, é de interesse do clínico e de profissionais especializados na caracterização da etiopatogenia e do desempenho nos casos em estudo.

Pacientes com distúrbios ou lesões que acarretam sobrecarga de pressão ou de volume e mesmo aqueles que evoluem com disfunção diastólica, podem se beneficiar com a obtenção de parâmetros obtidos pelo método de análise cardiopulmonar, também denominado ergoespirometria (TE-CP).

\section{Metodologia e interpretação de dados obtidos em avaliação cardiopulmonar ao esforço}

Indicaremos a seguir como interpretar os dados obtidos com a metodologia num verdadeiro check-list dos parâmetros em estudo.

Condições preliminares para uma boa avaliação - A ansiedade gerada na expectativa do exame, bem como a utilização de bucal próprio e o uso de clip nasal pelo indivíduo antes do início do teste, poderá eventualmente alterar o comportamento das variáveis ventilatórias. Portanto, é necessário um esclarecimento prévio da prova a ser feita e, em alguns casos, um treino com o sistema sem preocupação com o registro. Em nossa experiência, uma quantidade mínima de água deverá ser fornecida ao examinando antes do esforço a ser realizado, para ser evitado sensação desagradável de ressecamento com o uso do bucal.

Divisão de Medicina e Reabilitação - Instituto do Coração Hospital das Clínicas da Faculdade de Medicina da USP.

Correspondência: Paulo Yazbek Jr - Rua Pedroso Alvarenga, 333/42 - 04531-010 - São Paulo, SP

Recebido para publicação em 6/4/98

Aceito em 19/5/98
Os registros eletrocardiográficos com as derivações selecionadas são feitas previamente e as manobras ventilatórias, salvo estudo específico, não deverão ser utilizadas.

Pede-se ao paciente uma inspiração e expiração profunda com discretos movimentos de marcha estacionária por alguns segundos, observando o relaxamento muscular dos ombros e do tórax, evitando a respiração superficial. $\mathrm{O}$ início do teste deverá ser realizado, em geral, de 3 a $5 \mathrm{~min}$ após a introdução do bucal e clip nasal. Aguarda-se, para isto, $\mathrm{VE}, \mathrm{QR}$ e consumo de oxigênio $\left(\mathrm{VO}_{2}\right)$ adequados. A VE de repouso ideal para início do exercício situa-se entre $8 \mathrm{e}$ $15 \mathrm{~L} / \mathrm{min}, \mathrm{o} Q \mathrm{QR}$ entre 0,75 e 0,85 e o $\mathrm{VO}_{2}$ de repouso próximoa $3,5 \mathrm{~mL} / \mathrm{kg} / \mathrm{min}$, correspondente a $1 \mathrm{MET}$.

As condições de temperatura ambiente (próximo a $22^{\circ} \mathrm{C} \pm 2$ ), e umidade relativa do ar em torno de $60 \%$, seriam ideais no momento da prova.

No local, equipamentos de emergência (desfibriladore medicamentos) necessários para uma eventual parada cardíaca ou arritmia grave.

A calibração do equipamento, prévia ao exame, énecessária pois algumas variáveis são analisadas em presença de vapor d'água em condições denominadas de BTPS (body temperature pressure saturated), ex.: a VEque inclui a frequiência respiratória (FR) e o volume corrente (VC).

Outros parâmetros como o consumo de oxigênio $\left(\mathrm{VO}_{2}\right)$ e a produção de dióxido de carbono $\left(\mathrm{VCO}_{2}\right)$ são analisados em condições denominadas de STPD (standard temperature pressure and dry), que corresponde a situação de $\mathrm{O}^{\circ} \mathrm{C}$ de temperatura, pressão de $760 \mathrm{mmHg}$ ao nível do mar e em condições de ausência de vapor de água, ou seja, seco.

Nas relações que incluem a VE, $\mathrm{o} \mathrm{VO}_{2}$ ou $\mathrm{VCO}_{2}$, comoo equivalente ventilatório de $\mathrm{VO}_{2}\left(\mathrm{VE} / \mathrm{VO}_{2}\right)$, lê-se o numerador em condições de BTPS e o denominador em STPD, sendo da mesma maneira avaliada a relação $\mathrm{VE} / \mathrm{VCO}_{2}$.

É polêmica a discussão dos protocolos a serem empregados. Não existindo uma concordância, devemos empregar aquele que se adapte melhor ao caso. O protocolo de rampa tem sido muito utilizado, porém não podemos descartar o uso de avaliações que forneçam o que chamamos de steady-state ou equilíbrio de carga durante algum intervalo de tempo. Muito útil, quando queremos realmente saber se o paciente ou atleta encontra-se em condições aeróbias. É estipulado o tempo em torno de $12 \mathrm{~min}$ como necessário para uma boa eficácia de prova, caso não haja limitações por cardiopatia grave ${ }^{6}$. 


\begin{tabular}{|lll|}
\hline \multicolumn{1}{|c|}{$\begin{array}{l}\text { Quadro I - Escala de classificação de percepções subjetivas do nível } \\
\text { do esforço realizado e duas variações de terminologias utilizadas } \\
\text { para TE e programas de exercícios. } \\
\text { Índice de percepção de esforço de Borg }\end{array}$} \\
\hline & Variação (I) & Variação (II) \\
\hline $\begin{array}{l}\text { 6. } \\
\text { 7. Muito, muito leve }\end{array}$ & Muito, muito leve & Muito fácil \\
$\begin{array}{l}\text { 8. Muito leve } \\
\text { 10. }\end{array}$ & Muito leve & Fácil \\
$\begin{array}{l}\text { 11. Leve } \\
\text { 12. }\end{array}$ & Pouco leve & Relativamente fácil \\
$\begin{array}{l}\text { 13. Um pouco difícil } \\
\text { 14. }\end{array}$ & Um pouco forte & Ligeiramente cansativo \\
$\begin{array}{l}\text { 15. Difícil } \\
\text { 16. }\end{array}$ & Forte & Cansativo \\
17. Muito difícil & Muito forte & Muito cansativo \\
18. & Muito, muito forte & Exaustivo \\
19. Muito, muito difícil & M. & \\
\hline
\end{tabular}

Tabela para quantificar o esforço, como a de Borg (quadro I), é de fundamental importância pois complementa com dados objetivos a subjetividade declarada do esforço, bem como para orientar o examinador na indicação de exercícios adequados.

Obtenção de um teste verdadeiramente máximo $\left(\mathrm{VO}_{2} \max \right)$ - Pela ergometria convencional, a obtenção de frequiência cardíaca (FC) máxima, com a utilização de fórmulas (220-idade) ou (210-idade x 0,65), é sujeita a um desvio padrão de até $\pm 12 \mathrm{bpm}$, comprometendo muitas vezes o treinamento em pacientes que necessitam controle mais vigoroso pela presença de arritmia, hipertensão arterial, isquemia, etc.

Pelo uso da ergoespirometria é possível determinar, com relativa precisão, $\mathrm{o} \mathrm{VO}_{2}$ max com os seguintes dados: a) presença de $\mathrm{QR}\left(\mathrm{VCO}_{2} / \mathrm{VO}_{2}\right)>1.1$; b ) existência de um limiar anaeróbio (limiar de lactato); c) VE $>60 \%$ da máxima prevista; e) eventual presença de um platô no $\mathrm{VO}_{2}$ diante de um aumento na carga de esforço.

Estes dados, concomitantes à avaliação de FC atingida e a sensação subjetiva de esforço podem assegurar um teste máximo. Weber e $\mathrm{col}^{3}$ sugeriram que a classificação funcional dos pacientes portadores de insuficiência cardíaca congestiva (ICC) teria sido baseada nas respostas de limiar aeróbio (LA) e $\mathrm{VO}_{2}$ max. Os indivíduos que apresentam valores de $\mathrm{VO}_{2}$ noLA entre $11 \mathrm{e} 14 \mathrm{~mL} / \mathrm{kg}^{-1} \mathrm{~min}^{-1} \mathrm{eVO}_{2}$ max entre $16 \mathrm{e} 20 \mathrm{~mL} / \mathrm{kg}^{-1} \mathrm{~min}^{-1}$ foram considerados com ICC leve a moderada, enquanto os que tiveram valores de $\mathrm{VO}_{2}$ entre $5 \mathrm{e}$ $8 \mathrm{~mL} / \mathrm{kg}^{-1} \mathrm{~min}^{-1}$ noLAe $\mathrm{VO}_{2} \max <10 \mathrm{~mL} / \mathrm{kg}^{-1} \mathrm{~min}^{-1}$ foram classificados com ICC grave. Além disso, nesse estudo o $\mathrm{VO}_{2}$ max correlacionou-se estreitamente com o índice cardíaco (IC) durante o exercício. Pacientes com $\mathrm{VO}_{2} \max >20 \mathrm{~mL} / \mathrm{kg}^{-1}$ $\mathrm{min}^{-1}$ mostraram um IC $>8 \mathrm{Lmin}^{-1} \mathrm{~m}^{2}$, entretanto, aqueles com $\mathrm{VO}_{2} \max <10 \mathrm{ml} \mathrm{kg}^{-1} \mathrm{~mm}^{-1}$ mostraram um IC $<4 \mathrm{Lmin}^{-1} \mathrm{~m}^{2}$.

A relação $\Delta \mathrm{VO}_{2} / \mathbf{\Delta}$ WR é outra variável importante, medida durante exercício de protocolo de rampa, cujo valor incrementa-se progressivamente até o máximo; o valor normal é de cerca de $10 \mathrm{ml} / \mathrm{min} / \mathrm{W}$ quando um incremento de 10 a 20watt/min de rampa é empregado. Valores inferiores poderão significar uma baixa na função de reserva cardíaca.

Em 1985, Szlachcic e col ${ }^{6}$ verificaram que os pacientes com $\mathrm{VO}_{2}$ max $<10 \mathrm{~mL} / \mathrm{kg}^{-1} \mathrm{~min}^{-1}$ tinham mortalidade de $77 \%$ em um ano, e nos com $\mathrm{VO}_{2}$ max entre $10 \mathrm{e} 18 \mathrm{~mL} / \mathrm{kg}^{-1} \mathrm{~min}^{-1}$, esse índice diminuía para $14 \%$.

Convém lembrar que indivíduos adultos que apresentam valores $>40 \mathrm{~mL} / \mathrm{kg}^{-1} \mathrm{~min}^{-1}$ já apresentam algum tipo de condicionamento físico e os situados entre 20 a $40 \mathrm{~mL} / \mathrm{kg}$ ${ }^{1} \mathrm{~min}^{-1}$ são quase sempre sedentários não necessariamente portadores de cardiopatia.

Ventilação pulmonar (VE) - Resultante do produto da FR pelo VC. Fisiologicamente, durante o exercício, o incremento da VEé proporcional à produção de dióxido de carbono $\left(\mathrm{VCO}_{2}\right)$.

A VE, durante o TE-CP, aumenta progressivamente atingindo um platô máximo, caracterizando uma maior produção de $\mathrm{CO}_{2}$.

Em esforço, a VE poderá atingir até $200 \mathrm{~L}$ de ar ventilado por minuto (em atletas), sendo limitada em cardiopatas e pneumopatas. Como ela é resultante do produto FRxVC, a avaliação isolada destes dois parâmetros, muitas vezes, fazse necessária. A FR durante o teste, raramente, ultrapassa 50 ciclos/min, e o VC representa, parcialmente, a capacidade de expansibilidade pulmonar. O VC que, em repouso, pode variar de $300 \mathrm{a} 600 \mathrm{~mL}$ por movimento respiratório pode aumentar até, aproximadamente, $70 \%$ da capacidade vital ao esforço.

Alguns equipamentos fornecem dados da relação existente entre o espaço morto (VD) e o VC (ou VT-tidal volume). O comportamento normal do chamado VD/VT diminui durante o esforço em indivíduos normais. O incremento poderá significar modificações significativas na relação VE/ perfusão pulmonar.

Equivalentes respiratórios de $\mathrm{VO}_{2} \mathrm{eVCO}_{2}$ - As relações VE/ $\mathrm{VO}_{2} \mathrm{e} \mathrm{VE} / \mathrm{VCO}_{2}$, mantendo-se a VEem condições de BTPS e $\mathrm{VO}_{2}$ e VCO $\mathrm{em}_{2} \mathrm{STPD}$, relacionam quantos litros de ar por minuto são necessários e devem ser ventilados para consumir $100 \mathrm{~mL} \mathrm{de} \mathrm{O}_{2}$ (normal entre 2,3e $2,8 \mathrm{~L} / 100 \mathrm{~mL}$ ) ou produzir em $\mathrm{CO}_{2}$. Poderá esta relação ser expressa em 23 a 28 litros de ar ventilado para 1 litro de $\mathrm{O}_{2}$ consumido.

Durante o esforço crescente, as relações VE/ $\mathrm{VO}_{2} \mathrm{e} \mathrm{VE/}$ $\mathrm{VCO}_{2}$ diminuem, progressivamente, para depois aumentar até o final do esforço. A VE/ $\mathrm{VO}_{2}$ atinge valores mínimos precedendo a relação VE/ $\mathrm{VCO}_{2}$ Âs variáveis citadas são de fundamental importância na detecção do limiar anaeróbio (LA) como veremos adiante. (fig. 2).

Pressão expirada de $\mathrm{O}_{2}\left(\mathrm{PETO}_{2}\right)$ ou fração expirada de $\mathbf{O}_{2}\left(\right.$ FEO $\left._{2}\right)-$ A PETO $_{2}$ em repouso é de $\pm 90 \mathrm{mmHg}$, diminui transitoriamente logo após o início do exercício, desde que o aumento na $\mathrm{VE}$ seja mais lento que o incremento no $\mathrm{VO}_{2}$. Ao ultrapassar o LA I, a $\mathrm{PETO}_{2}$ aumenta 10 a $30 \mathrm{mmHg}$ ao atingir o esforço máximo, devido a hiperventilação provocada pela diminuição do $\mathrm{PH}$. $\mathrm{A} \mathrm{FEO}_{2}$ tem o mesmo comportamento, diminuindo no início do esforço e atingindo um valor mínimo, incrementando-se a seguir. Este parâmetro facilita a detecção do limiar anaeróbio I (LA I). 
Pressão expirada de dióxido de carbono $\left(\right.$ PETCO $\left._{2}\right)-\mathrm{O}$ valor da PETCO $_{2}$ ao nível do mar varia de 36 a $42 \mathrm{mmHg}$. Eleva-se 3 a $8 \mathrm{mmHg}$ durante exercício de intensidade leve a moderada, atinge um máximo, caracterizando o LAII, e pode em seguida diminuir. $\mathrm{AFECO}_{2}$ tem o mesmo comportamento durante exercícios de carga crescente.

$\mathrm{QR}$ e/ou razão de troca respiratória (RER-R) $-\mathrm{QR}=$ $\mathrm{VCO}_{2} / \mathrm{VO}_{2}$-Esta variável significa relação entre $\mathrm{o} \mathrm{CO}_{2}$ produzidoe o $\mathrm{O}_{2}$ consumido. Aorealizar exercício com R próximo de 0,70 , estamos consumindo mais lipídeos. Com valores próximos de 1,00, consumimos mais carboidratos. Exemplos:

$\mathrm{C}_{6} \mathrm{H}_{12} \mathrm{O}_{6}+6 \mathrm{O}_{2}=6 \mathrm{CO}_{2}+\mathrm{H}_{2} \mathrm{O}+\mathrm{E}-$ portanto $\mathrm{QR}=6 \mathrm{CO}_{2} / 6 \mathrm{O}_{2}=1$; (glicose)

$\mathrm{C}_{16} \mathrm{H}_{32} \mathrm{O}_{24}+\mathrm{nO}_{2}=6 \mathrm{CO}_{2}+\mathrm{H}_{2} \mathrm{O}+\mathrm{E}$-portantoQR $=6 \mathrm{CO}_{2} / \mathrm{nO}_{2}=<1,0$ (ex. de lípides)

Durante a combustão de carboidratos, forma-se $830 \mathrm{~cm}^{3} \mathrm{de}$ $\mathrm{CO}_{2}$ com $1 \mathrm{~g}$ delípidese $1430 \mathrm{~cm}^{3} \mathrm{deCO}_{2}$. Ovalorde $n$ serásempre incrementado para a produção do $\mathrm{CO}_{2} \mathrm{e}$, portanto, com gasto energéticomaior, caracterizandoum $\mathrm{QR}$ menor doque aunidade.

Pulso de oxigênio $\mathrm{PO}_{2}\left(\mathrm{VO}_{2} / \mathbf{F C}\right)$ - Considerada uma das mais importantes variáveis utilizadas pela ergoespiro- metria, pois demonstra a quantidade de $\mathrm{O}_{2}$ que é transportada a cada sistole cardíaca. Uma diminuição do desempenho de VE pode ser detectada em uma prova de esforço crescente com observação do pulso de $\mathrm{O}_{2}$.

Medição não invasiva do limiar anaeróbio e sua relação com a produção láctica. Importância na medição do desempenho - Desde que foi publicado o trabalho de Wasserman e $\mathrm{col}^{5}$ tem-se levantado muitas indagações sobre o conceito de LA entre os fisiologistas de exercício e bioquímicos. Porém hoje é bem definido o que ocorre durante um teste de esforço com cargas crescentes.

O ácido láctico é o ácido fixo predominante produzido durante exercício. Possui um $\mathrm{pH}$ de, aproximadamente, 3,8 e, portanto, está totalmente dissociado do $\mathrm{pH}$ da célula $(7,0)$. $\mathrm{O}$ sistema de tamponagem do $\mathrm{HCO}_{3}$ restringe a alteração do $\mathrm{pH}$, que ocorreria através da formação deste ácido relativamente forte, porque a reação: $\mathrm{H}^{+}+\mathrm{HCO}_{3} \leftrightarrow \mathrm{H}_{2} \mathrm{CO}_{3} \leftrightarrow \mathrm{CO}+$ $\mathrm{H}_{2} \mathrm{O}$ ou LaH$+\mathrm{NaHCO}_{3} \leftrightarrow \mathrm{NaLa}+\mathrm{CO}_{2}+\mathrm{H}_{2} \mathrm{O}$ se dirige à direita para formar $\mathrm{CO}_{2}$. Em associação com a formação $\mathrm{HCO}_{3} \mathrm{du}-$ rante a tamponagem, a concentração de $\mathrm{HCO}_{3}$ diminui em proporção inversa com o aumento na concentração de lactato. Em razão de a tamponagem do $\mathrm{H}^{+}$associada à produção de lactato ocorrer no meio intracelular, a produção de

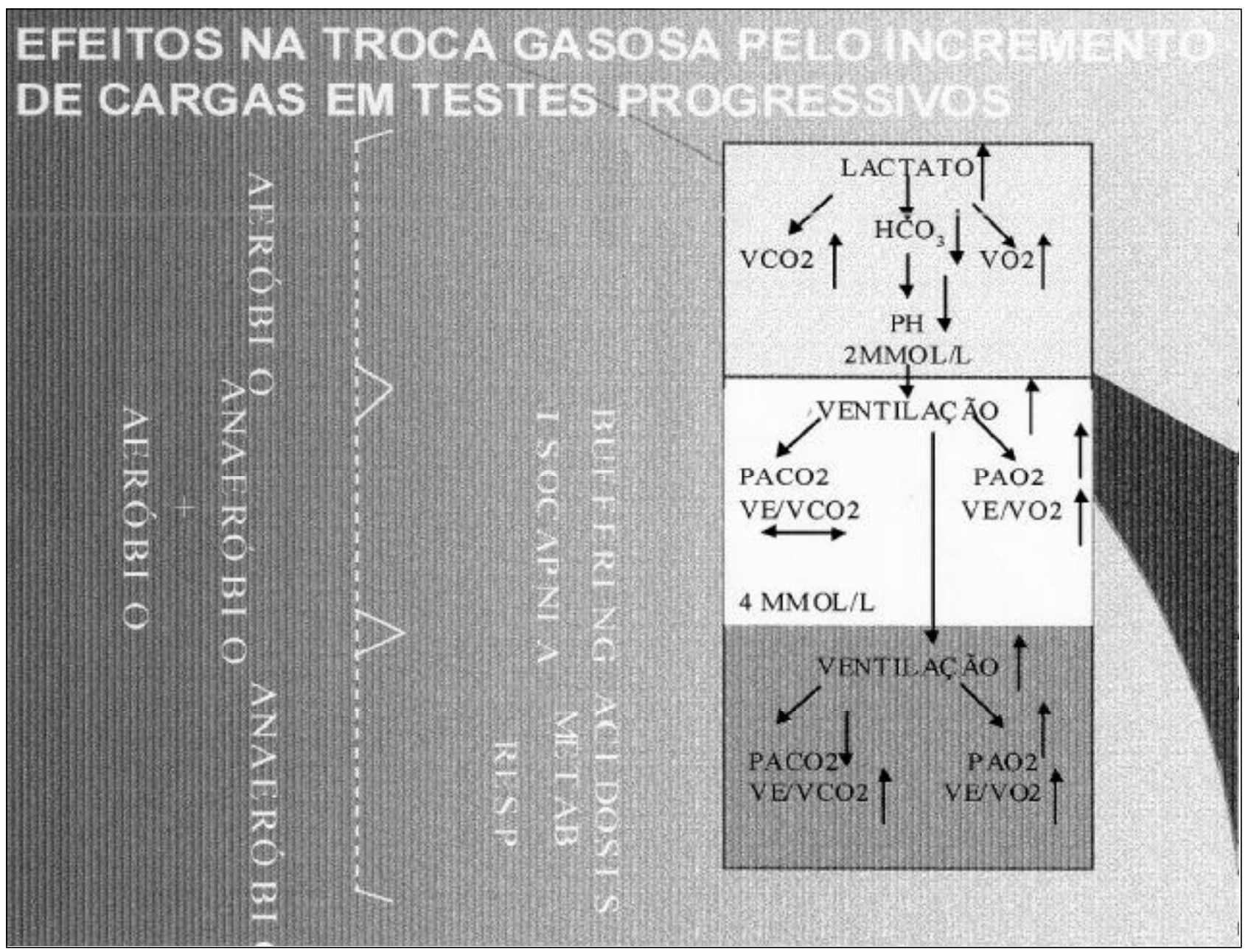

Fig. 1 - Comportamento metabólico durante esforço progressivo com incremento de cargas. 
$\mathrm{CO}_{2}$ pela célula deve aumentar. $\mathrm{O}$ aumento de lactato e a diminuição do $\mathrm{HCO}_{3}$ na célula serão rapidamente equilibrados, através de intercâmbio transmembrânico desses íons. Conseqüentemente, o fluxo do $\mathrm{CO}_{2}$ adicional, gerado na célula através da tamponagem, deverá ser detectado rapidamente na troca de gases do pulmão.

Um teste de nível de esforço progressivo, em que são medidas as trocas do gás, e o $\mathrm{VO}_{2}$ no LA, permite a medição dos fenômenos associados à acidose metabólica em desenvolvimento. À medida que aumenta o nível de esforço, $\mathrm{VO}_{2}$,
$\mathrm{VCO}_{2}$ e VEaumentam de forma linear. Acima do LA, a produção de ácido láctico acarreta um aumento de $\mathrm{CO}_{2}$ da célula e da taxa de $\mathrm{CO}_{2}$ venosa, o que resulta em aceleração do incremento de $\mathrm{VCO}_{2}$, geralmente acompanhado de um aumento paralelo em VE, mantendo, desta forma, o $\mathrm{PaCO}_{2}$ constante. Como o nível de incremento de $\mathrm{VO}_{2}$ permanece linear, enquanto o VE acelera, o $\mathrm{PETO}_{2}$ aumenta caracterizando o LA I enquanto o $\mathrm{PETCO}_{2}$ não diminui de forma recíproca. Estes fenômenos determinam o limiar I. Como corolário, o equivalente ventilatório para $\mathrm{O}_{2}\left(\mathrm{VE} / \mathrm{VO}_{2}\right)$ aumenta sem que haja

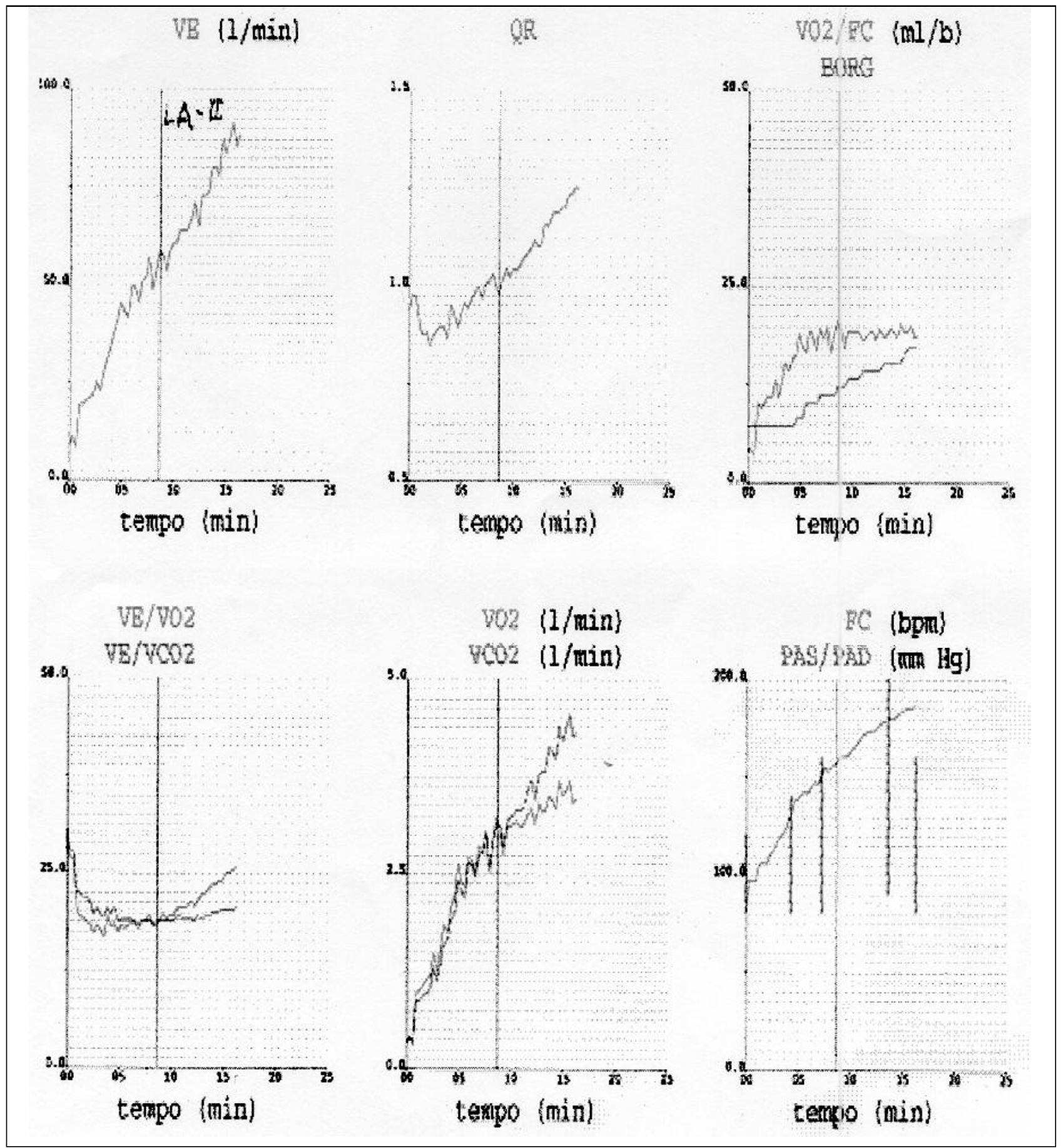

Fig. 2 - Comportamento das principais variáveis durante prova ergoespirométrica. 
um aumento no equivalente ventilatório para $\mathrm{CO}_{2}(\mathrm{VE} /$ $\mathrm{VCO}_{2}$ ). O estreito aumento paralelo em $\mathrm{VEe} \mathrm{VCO}_{2}$, visto inicialmente acima do LA, reflete um breve período de tamponagem isocápnica, istoé, $\mathrm{VE} / \mathrm{VCO}_{2}$ e PETCO $\mathrm{C}_{2}$ não se alteram, enquanto $\mathrm{VE} / \mathrm{VO}_{2}$ e $\mathrm{PETO}_{2}$ aumentam. De acordo com Wasserman e col, esta é uma demonstração sensível de troca de gás para a medição não-invasiva do limiar anaeróbio. À medida que o nível de esforço aumenta, o pH cai subseqüentemente, fazendo com que a VEaumente mais depressa do que a produção de $\mathrm{CO}_{2}$. Isto faz com que o $\mathrm{PaCO}_{2}$ caia e o $\mathrm{pH}$ aumente. Esta compensação respiratória para a acidose láctica não-respiratória resulta em um aumento de $\mathrm{VE} / \mathrm{VCO}_{2}$ bem como em um decréscimo adicional em $\mathrm{VE} / \mathrm{VO}_{2}$ (fig. 2), caracterizando o limiar II).

Quandoé medido no LA, o consumo de $\mathrm{O}_{2}\left(\mathrm{VO}_{2}\right)$ nãoé afetado pelo tipo de protocolo de exercício usado para uma determinada forma de esforço. Além do mais, $\mathrm{o} \mathrm{VO}_{2}$ no LA não será afetado pela duração de cada incremento de nível de esforço.

O limiar ventilatório aeróbio (LV 1) foi considerado como sendo o ponto em que houve quebra de linearidade do $\mathrm{VE} / \mathrm{VO}_{2}$, tendência de ascensão abrupta da razão de troca respiratória (RER) e menor pressão expirada final de oxigênio $\left(\mathrm{PETO}_{2}\right)$ ou fração expirada de $\mathrm{O}_{2}\left(\mathrm{FEO}_{2}\right)$. Olimiar ventilatório anaeróbio (LV2) foi considerado como o ponto em que houve quebra de linearidade do $\mathrm{VE} / \mathrm{VCO}_{2}$ e maior pressão expirada final de $\mathrm{CO}_{2}\left(\mathrm{PETCO}_{2}\right)$ ou fração expirada de $\mathrm{CO}_{2}\left(\mathrm{FECO}_{2}\right)$, precedendo sua queda abrupta. OLV 2 é também denominado ponto de descompensação ácido-metabólico (fig. 2).

OLA também pode ser determinado pelo método do $V$ slope detectado no chamado turning point da curva $\mathrm{VCO}_{2} \mathrm{x}$ $\mathrm{VO}_{2}$ (fig.3).

Boucher e $\mathrm{col}^{8}$, estudando indivíduos saudáveis, verificaram o comportamento da função ventricular, analisando o comportamento da FE do repouso até o LA e, deste, até a intensidade máxima de exercício. A FE aumentou do repouso para o LA, mas a mesma resposta não foi verificada do LA para o exercício máximo. Eles concluíram que o maior incremento da FE ocorre em estágios de esforço aquém do LA, sendo que, após, a resposta pode ser variável e um aumento uniforme não é necessariamente esperado em indivíduos normais. Em relação ao comportamento do desempenho ventricular durante o esforço, em relação ao LA, merece destaque o estudo de Goodman e $\operatorname{col}^{9}$ que concluiu que o

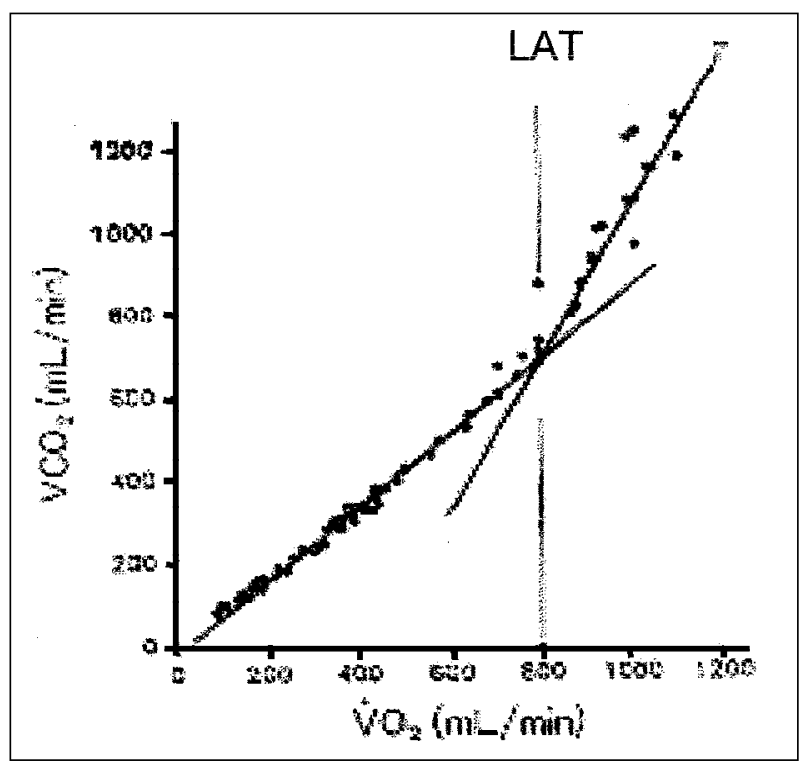

Fig. 3 - Determinação do limiar anaeróbio pelo método $V$-slope. O limiar anaeróbio é deteminado pela intersecção de 2 tangentes traçadas a partir da curva de ascenção do $\mathrm{VO}_{2}$ relativamente ao $\mathrm{VO}_{2}$ (turning point).

mecanismo de Frank/Starling exerce funções progressivamente diferente antes e após o LA. Os resultados sugerem que antes do LA, o mecanismo de Frank/Starling é mais operante e a contatilidade miocárdica, ao contrário, é mais efetiva acima do $\mathrm{LA}^{10,11}$

A importância na detecção do LA, para o clínico, incide no fato de que exercícios realizados numa intensidade acima do LA pode provocar um aumento abrupto nos níveis de catecolaminas, causando as conseqüências conhecidas de arritmia, hipertensão e isquemia do miocárdio.

Num estudo realizado no Instituto do Coração (InCor) do Hospital das Clínicas da FMUSP ${ }^{11}$, foram avaliados pacientes com ICC e os resultados apontaram comportamentos díspares nas variáveis ergoespirométricas, caracterizando capacidade funcional diminuída em pacientes que foram a óbito em tempo de esforço físico, em condições anaeróbias, menor que outros que não mostraram melhor desempenho.

Em conclusão, o método de avaliação com gases expirados apresenta-se como mais uma arma na propedêutica não invasiva de determinar a real capacidade funcional de atletas e pacientes com comprometimento cardiovasculare ou pulmonar.

\section{Referências}

1. The Criteria Committee of the New York Heart Association - Revision to classification of functional class and objective assessment of patients with disease of the heart. Circulation 1994; 92: 644-5.

2. Wasserman K, Hansen JE, Suedy, Whipp BJ - eds: Principles of Exercise Testing and Interpretation. Philadelphia: Lca and Febiger, 1987; 47-57.

3. Weber K, Janicki JS - Cardiopulmonary exercise testing for evaluation of chronic heart failure. Am J. Cardiol. 1985; 55: 22A-31A.

4. Van den Brock SHJ, Van Veldhuisen DJ, de Graess PH, Landsman MLJ, Hillege H, Lie KI - Comparison between New YorK Heart Association classification and peak oxygen consumption in the assessment of functional status and prognosis in patients with mild to moderate chronic congestive heart failure secondary to either ischemic or idiopathic dilated cardiomyopathy. Am J Cardiol 1992; 359-63.

5. Wasserman K, Mcilroy MB - Detecting the threshold of anaerobic metabolism in cardiac patients during exercise. Am J Cardiol 1964; 14: 844-52.

6. Buchfuhrer MJ, Hansen JE, Robinson TE, Sue DY, Wasserman J, Whipp BJ Optimizing the exercise stress test (or cardiopulmonary assessment): J Appl Physiol 1983; 55: 1558-64.

7. Szlachac J, Massic DM, Kramer BL, Topic N, Tabau J - Correlates and prognostic 
implication of exercise capacity in chronic congestive heart failure. Am J Cardiol $1985 ; 1037-42$.

8. Boucher CH, Andersen MD, Schkeider MS, Marphy JH, Okada RD, Kanarck DJ - Left ventricular function before and after reaching the anaerobic threshold. Chest, 1985; 87: 145-50.

9. Goodman JM, Pluley MJ, Leskowitz CH, Lia PP, Mclaughtr PR - Left ventricular functional response to moderate and intense exercise. Am J Spt Sci 1991; 16: 104-9.
10. Wasserman K, Beauck WL, Whipp BJ - Gas exchange theory and the lactic acidosis (anaerobic) threshold. Circulation 1990; (supp II): II 14-II 30

11. Franciosa JH. Park M, Levine TB - Lack of correlation between exercise capacity and indexes of resting lest ventricular performance in heart failure. Am J Cardiol 1981; 47: 33-47.

12. Paulo Yazbek Jr, Linamara Rizzo Battistella - Condicionamento Físico do Atleta ao Transplantado. Aspectos Multidisciplinares na Prevenção e Reabilitação Cardíaca. São Paulo: Savier: APM - 1994 